$\xi=$ 줄

\title{
Papillon lefevre syndrome- An imperative role of periodontist
}

\author{
Prathypaty Santha Kumari ${ }^{1}$, Guvvala Satheesh Kumar $^{2 *}$, Potharaju Santhi Priya ${ }^{3}$, \\ Yendluri Durga Bai ${ }^{4}$, Sahithi Dathar ${ }^{5}$ \\ ${ }^{1}$ Professor and Head, Department of Periodontology, Government Dental college and hospital, Hyderabad \\ ${ }^{2}$ Post graduate student, Department of Periodontology, Government Dental college and hospital, Hyderabad \\ ${ }^{3}$ Assistant Professor, Department of Periodontology, Government Dental college and hospital, Hyderabad \\ ${ }^{4}$ Associate Professor, Department of Periodontology, Government Dental college and hospital, Hyderabad \\ ${ }^{5}$ Post graduate student, Department of Oral medicine and Radiology, Vishnu Dental College, Bhimavaram \\ *Corresponding author E-mail: satheesh.guvvala@gmail.com
}

\begin{abstract}
Papillon Lefevre syndrome (PLS) is a rarely encountered disorder that shows autosomal recessive inheritance. Clinically, it exhibits hyperkeratotic plaques on palmar plantar surfaces, severe periodontal loss and precocious shedding of primary as well as permanent dentition. The reason for this exceptional clinical presentation is vague and speculative. We are reporting one such case of a $23 y$ ear old female patient.
\end{abstract}

Keywords: Papillon Lefevre Syndrome; Periodontist; Socket Preservation

\section{Introduction}

Papillon Lefevre syndrome (PLS) is a very rare entity inherited as an autosomal recessive disorder (Hegde R et al. 2002, Dhadke et al. 2006, Pavankumar et al. 2010, Kansky et al.). Its prevalence is about 1 to 4 persons per million and shows no sex predilection as well as no racial predominance (Pavankumar et al. 2010) However, a genetic predisposition exists with greater chance of occurrence in the consanguineous offspring (Singla et al. 2010, Kaur et al. 2013). The syndrome is characterised by palmarplantar hyperkeratosis, severe periodontitis, and premature exfoliation of primary and permanent dentition. The hyperkeratotic plaques are sharply demarcated and more frequently show an extensive involvement of the entire surface of palms and soles (Pavankumar et al. 2010). Well-distinguished psoriasiform plaques are also represented on elbows and knees.

The next recognised feature is severe periodontitis which results in marked attachment loss and severe alveolar bone destruction eventually leading to early loss of dentition (Dorairaj et al. 2012). The eruption of the deciduous teeth is normal, but they soon tend to exfoliate and the gingiva appears to be as healthy as before. Nevertheless, the eruption of permanent teeth again triggers the aggressive periodontitis, which results in partial or complete loss of teeth at a very young age (Sachdeva et al. 2012). The periodontist therefore play a very essential role in early management of this severity so as to preserve the teeth and probably gain a predictable outcome.

It has been reported in literature that $20-25 \%$ of patients show an increased susceptibility to infections, commonly otitis media and thus patient also suffer from hearing difficulties (Kaya et al. 2008). Therefore, multidisciplinary approach is of utmost importance in early diagnosis and management of this disease. Herewith we are reporting one such rare case of a 23 year old young female.

\section{Case report}

A 23 year old female patient has reported to the Department of Periodontics, Government Dental College and Hospital, Hyderabad with a chief complaint of loosening of teeth since 8 years. She gave history of early loss of primary dentition at age about 4-5 years and delayed eruption of permanent teeth. She also gives history of bleeding gums that gets aggrevated on brushing and chewing food. Medical history revealed recurrent scaling of her skin especially palms and soles that worsens in winter. Family history had revealed consanguineous marriage of her parents and no other family members had similar presentation. Patient underwent prosthetic rehabilitation of her upper anterior teeth 4 years back.

On examination, all her vital signs were within the normal range. Severe palmar plantar hyperkaratotis with well demarcated, rough, yellowish plaques were observed bilaterally and peeling of the superficial skin from the palms and soles was apparent. Mild demarcated keratotic plaques were also noticed involving the elbows as depicted in figure 1 .

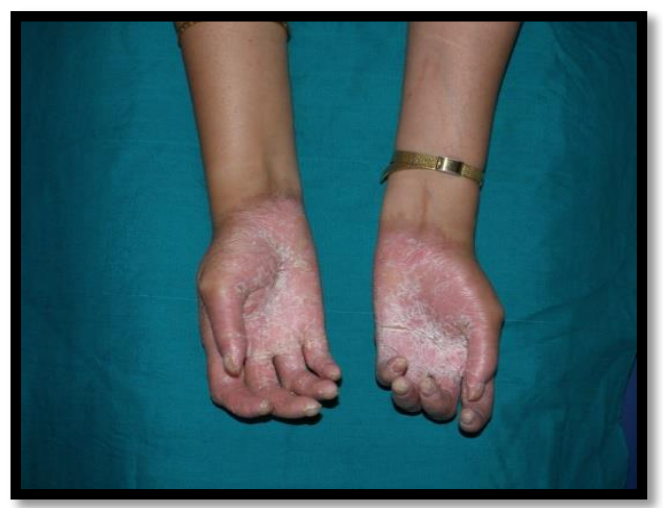



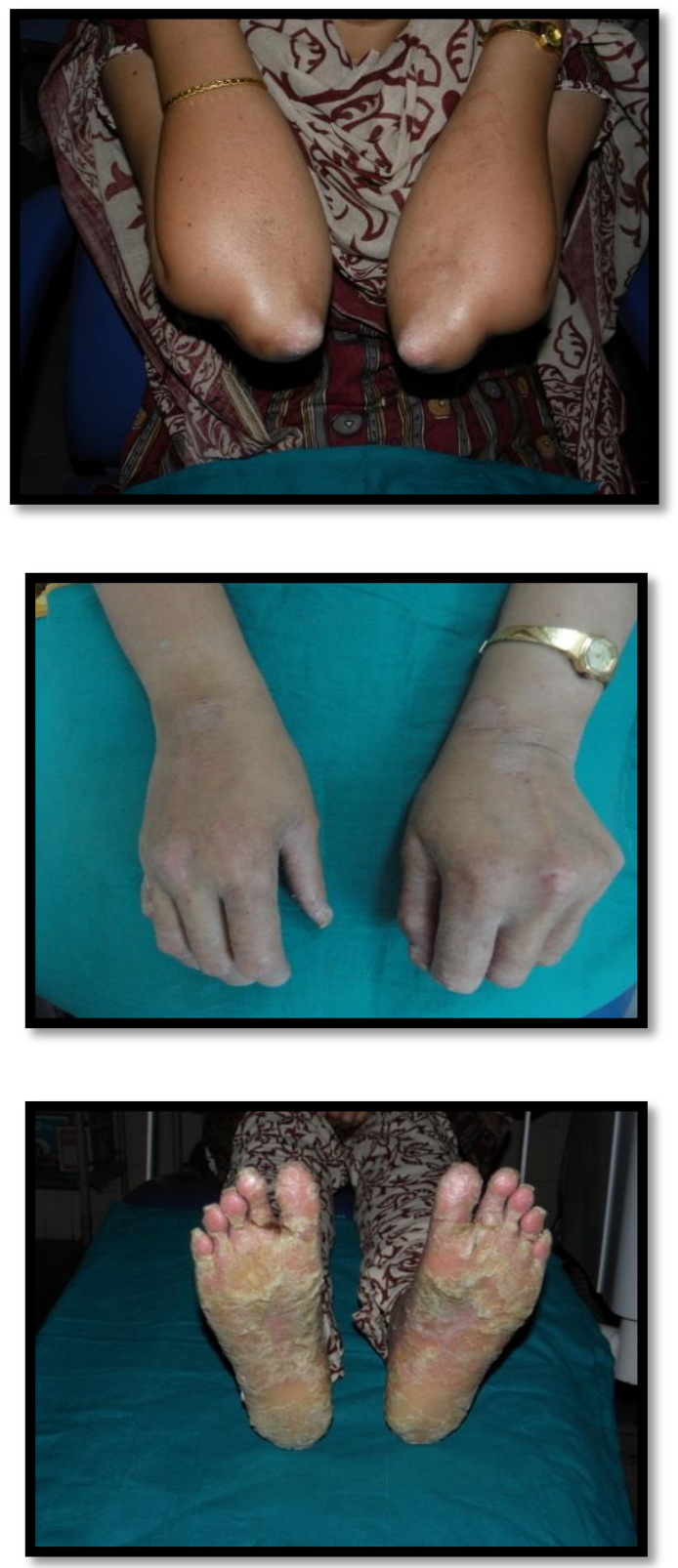

Fig. 1: Palmar-Plantar Hyperkeratosis.

Intraoral examination revealed generalised gingival recession (figure 2) and generalised grade I mobility of the teeth. Grade III mobility was observed with respect to 44 and was thus advised for extraction of 44. Fixed prosthetic bridge was evident in the anterior maxillary region.

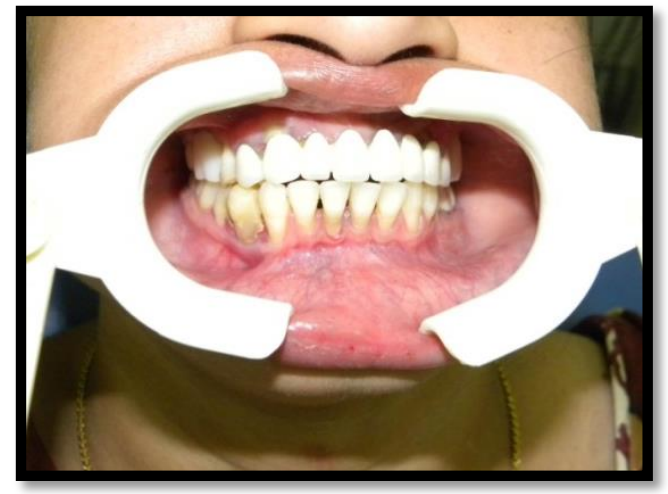

Fig. 2: Severe Periodontitis.

The periondontist approach had mainly included the complete oral prophylaxis by scaling and root planning followed by socket preservation in relation to the extracted 44(figure 3). Patient was emphasized on oral good oral hygiene reinforcement and was advised for regular recall checkups for every 3 months. Patient was referred to a Dermatologist for further needful palliative treatment.

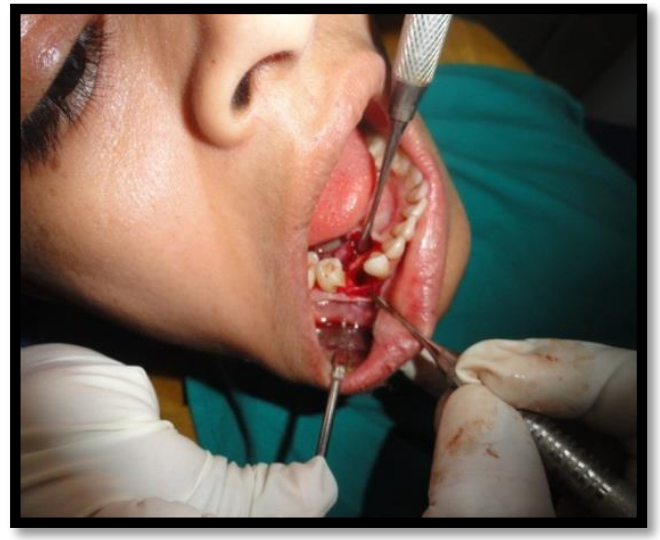

Fig. 3: Socket Preservation.

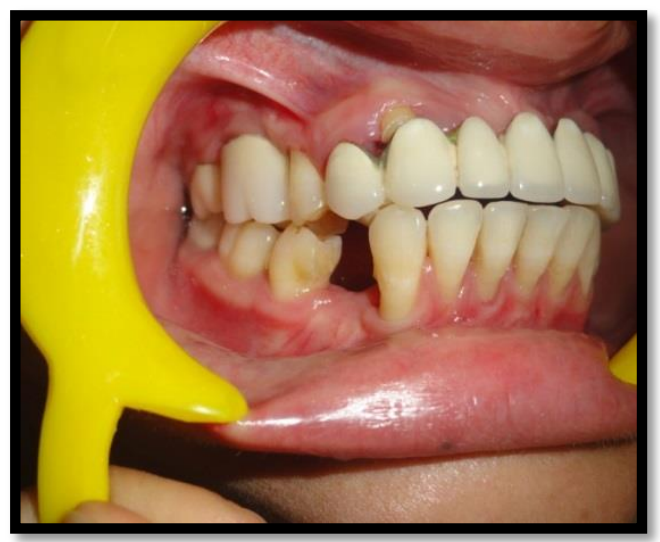

Fig. 4: Post-Surgical.

\section{Discussion}

Papillon Lefevre syndrome is an unusual entity which is inherited as an autosomal recessive disorder. Though it shows a negative family history, it can occur in other siblings along with the effected individual (Singla et al. 2010). It was first described by two French physicians Papillon and Lefevre and hence the name was proposed accordingly (Singla et al. 2010, Kola et al. 2014).

The underlying etiology is still unknown and obscure. Yet recent studies have proposed that cathepsin $\mathrm{C}$ gene mutation localized to chromosome $11 \mathrm{q} 14-21$ is responsible for the occurrence of this disorder. The expression of cathespin $\mathrm{C}$ is known to be evident in the neutrophils and epithelial regions such as soles, palms and keratinised mucosa like gingiva and it is functionally significant in structural growth and development of skin and also in susceptibility to occurrence of periodontal disease (Singla et al. 2010, Kaur et al. 2013, Kumar et al. 2014). Therefore, the characteristic features exemplified in this syndrome include palmar plantar hyperkeratosis, severe loss of periodontium around the teeth and due to the loss of this supporting structure it ultimately results in early loss of dentition. The precocious loss of deciduous teeth is noticed at the age of 3-4 years (Dorairaj et al. 2012). Severe resorption of alveolar bone shows characteristically the teeth as 'floating-in-air' appearance on panoramic radiographs (Pavankumar et al. 2010). Gorlin et al. have added the third feature of dural calcification (Shafi et al. 2013). Although this can be taken as a unique feature, but it is inconsistent and thus not considered mandatory for the diagnosis. 
The differential diagnosis can probably include hypophosphatasia. Clinically knock-knee, bowing of femur and tibia and enlarged wrists are observed and intraorally premature shedding the teeth is evident. Diagnosis is based on increased phosphor-ethanolamine levels in urine (Singla et al. 2010). Another condition to be distinguished is cyclic neutropenia which may present with severe periodontal disease but palmoplantar hyperkeratosis is absent.

The history of the present case had elucidated seasonal variations in the scaling of the skin that worsens in winter which is similar to the cases reported by Singla et al (2010), and Sachdeva et al (2012).

The essential role of periodontist mainly lies in elimination of the reservoir containing the offending bacteria by means of complete oral prophylaxis. The extraction of teeth should be immediately followed by socket preservation techniques so as to conserve the socket. Other periodontal surgical treatments like flap surgery with bone grafting can be implicated in severely affected individuals. The patients should also be advised for good oral hygiene maintenance and regular recall examinations. The management of PLS also requires a multidisciplinary approach with the active participation of the dental surgeon and dermatologist (Singla et al. 2010).

Early diagnosis is of utmost importance so as to employ prompt management and ultimately showing considerable benefit to the patient.

\section{Conclusion}

Papillon Lefevre syndrome is an uncommon inherited disorder which shows both cutaneous and oral involvement affecting the social well-being of the patient. The dental surgeons and the dermatologist should be aware of this unusual entity so as to diagnose and manage promptly and accordingly. The periodontist does play a significant role preservation of the teeth by employing various recommended surgical techniques required for the affected individuals and should also consider the psychological well-being of the patients so as to benefit the mankind.

\section{References}

[1] Pavankumar (2010). Papillon Lefevre syndrome - A case report. The Saudi Dental Journal. 22, 95-98. http://dx.doi.org/10.1016/j.sdentj.2010.02.009.

[2] Dhadke et al (2006). Papillon Lefevre syndrome. JAPI.54:246-247

[3] Kaya FN et al (2008). International Dental and Medical Disorders 1: $24-28$

[4] Dorairaj et al (2012). Papillon Lefevre syndrome-A periodontist approach. International Journal of Case Reports and Images. 3 (7):15 http://dx.doi.org/10.5348/ijcri-2012-07-140-CS-1.

[5] Singla A, Sheikh S, Jindal SK, Brar R (2010). Papillon Lefevre syndrome: Bridge between Dermatologist and Dentist J Clin Exp Dent. 2(1):e43-6.

[6] Sachdeva et al (2012).Papillon- Lefèvre Syndrome:Report of a case and its management J Clin Exp Dent. 4(1):e77-81. http://dx.doi.org/10.4317/jced.50594.

[7] Kaur et al (2013). Papillon Lefevre Syndrome: A Case Report with Review. Dentistry. 3(1):1-4 http://dx.doi.org/10.4172/21611122.1000156

[8] Hegde R et al (2002). Papillon Lefevre Syndrome: a report of two cases. Indian Sot Pedo Prev Dent. 20:1:9-11.

[9] Kumar et al (2014). Papillon Lefevre Syndrome - A Brief Review of Diagnosis \& Management. Journal of Dental and Medical Sciences; 13(11):92-95. http://dx.doi.org/10.9790/0853-131169295.

[10] Kansky et al., Papillon Lefevre Syndrome. Acta dermatovenerologica:6(2);60-65

[11] Kola MZ (2014). Prosthodontic Management of Papillon-Lefevre syndrome. Journal of Advanced Medical and Dental Sciences Research.2 (4):97-104.

[12] Shafi et al (2013). Papillion Lefevre Syndrome-A Case Series of Siblings. Sch. J. App. Med. Sci. 1(5):589-592. 